\title{
Clostridium difficile infection in patients with inflammatory bowel disease
}

\author{
Jacek Czepiel ${ }^{1}$, Grażyna Biesiada ${ }^{1}$, William Perucki², Tomasz Mach ${ }^{1}$ \\ ${ }^{1}$ Department of Gastroenterology, Hepatology and Infectious Diseases, Jagiellonian University, Medical College, Krakow, Poland \\ ${ }^{2}$ Students' Scientific Society, Jagiellonian University, Medical College, Krakow, Poland
}

Prz Gastroenterol 2014; 9, 3: 125-129 DOI: $10.5114 / p g .2014 .43572$

Key words: Clostridium difficile, inflammatory bowel disease.

Address for correspondence: Jacek Czepiel MD, PhD, Department of Infectious Diseases, Jagiellonian University, Medical College, 5 Śniadeckich St, 31-501 Krakow, Poland, phone/fax: +48 1242473 49, e-mail: jacek.czepiel@uj.edu.pl

\begin{abstract}
Clostridium difficile is a bacterium widely distributed in the human environment. In the last decade the incidence and severity of Clostridium difficile infection has grown, particularly in Europe and North America, making it one of the more common nosocomial infections. A group particularly susceptible to Clostridium difficile infection are patients with inflammatory bowel disease, especially those with involvement of the colon. This paper presents relevant data on Clostridium difficile infections in inflammatory bowel disease patients, including epidemiology, pathogenesis, diagnosis and treatment.
\end{abstract}

\section{Introduction}

Clostridium difficile is a gram positive, spore-producing anaerobic rod. It was first isolated by Hall and O'Toole in 1935 [1]. In 1974, Tedesco et al. reported diarrhoea in $21 \%$ of patients treated with clindamycin, with half of them displaying pseudomembranes on endoscopy [2]. The set of symptoms caused by Clostridium difficile infection is referred to as either Clostridium difficileassociated disease (CDAD) or Clostridium difficile Infection (CDI), and these terms are often used interchangeably. In the last decade there was an increase in both the frequency and severity of CDI. It is more common in hospitalised patients; hence patients hospitalised with inflammatory bowel disease (IBD) are a particularly vulnerable group. The CDI infection in these patients occurs more frequently and often runs a more severe course than observed in the general population $[3,4]$.

\section{Epidemiology}

The incidence of CDI in patients with IBD depends the amount of the GI tract consumed by, as well as the extent of, the disease. Ricciardi et al. published the largest existing cohort study, which followed more than 350,000 patients with IBD over 10 years in the United States. The study showed CDI in $2.8 \%$ of patients with ulcerative colitis (UC), and in $1 \%$ of patients with Crohn's disease (CD) [5]. Nguyen et al. evaluated CDAD incidence in patients hospitalised between 1998 and 2004 and noted that the incidence of CDI in patients with UC was $3.73 \%$, in patients with CD it was $1.09 \%$ while only $0.45 \%$ of patients in the general population became infected with CDI. Additionally, a two-fold increase in the incidence of CDAD in patients with UC, from $2.66 \%$ to $5.12 \%$, was noted during seven years of observation [6]. Issa et al. found that the incidence of CDAD in patients with IBD increased from $1.8 \%$ in 2004 to $4.6 \%$ in 2005 , and patients with IBD involving the colon tended to be infected more frequently [7, 8]. Ananthakrishnan et al. found patients with IBD and CDAD were hospitalised more frequently, and that the frequency grew over time from 24/1000 cases in 1998 to $39 / 1000$ cases in 2004 for UC patients and 8/1000 in 1998 to 12/1000 in 2004 for CD patients [9].

Thus, the last decade has seen an increase in the frequency and severity of CDAD in patients with IBD. The CDI is more common among patients with UC and $C D$ when compared to the general population. The risk of disease is higher in adults with UC than among those with CD. Such differences were not observed in children $[10,11]$. 


\section{Risk factors}

The IBD is an independent risk factor for CDI, with an approximately 3-fold increased risk compared with non-IBD patients. Proven risk factors for CDAD in patients with IBD include older age, as well as antibiotic or steroid use. The most significant factor in the development of CDAD in patients with IBD is antibiotic therapy. Approximately $60 \%$ of patients with IBD report the use of antibiotics prior to the development of CDAD. Many antibiotics can lead to the development of CDAD; however, clindamycin, third-generation cephalosporins, fluoroquinolones and broad-spectrum penicillins seem to be most frequently implicated [12]. Another risk factor is treatment with glucocorticoids. In a large cohort study in 2009, Schneeweiss et al. showed a three-fold higher risk of CDAD in patients with IBD treated with steroids, with no correlation between dose and duration of use [13]. Studies on other immunosuppressants and immunomodulators, including azathioprine, 6-mercaptopurine, methotrexate and infliximab, are ambiguous or have only been carried out in small groups [4]. Old age is also a risk factor in patients with IBD, though the average age of developing CDAD is lower for IBD patients than in the general population. One study of 51 patients with IBD showed that patients with more extensive involvement of the colon had a higher risk of developing CDAD. Additional research is needed to investigate this issue [14]. Data is lacking concerning the prevalence of Clostridium difficile BI/NAP1/027, a particularly virulent form of Clostridium difficile characterised by a severe course, in patients with IBD. One study assessed the frequency of specific strains of $C D$ in patients with clinical remission of UC or CD and demonstrated that of the seven toxin-producing ribotypes, only R015 was common to both diseases [15].

\section{Pathogenesis}

Virulence factors produced by Clostridium difficile include enzymes such as collagenase, hyaluronidase, chondroitin-sulphatase and, most importantly, toxins. The most important in the pathogenesis of CDI are toxins $A$ and $B$, which are both enterotoxic and cytotoxic, although traditionally toxin $A$ is defined as the enterotoxin and toxin $B$ is identified as the cytotoxin. The production of these toxins is regulated by the following genes: $t c d A, t c d B, t c d C, t c d D$ and $t c d E$ [3]. Some strains of Clostridium difficile produce a third toxin called binary toxin, whose role is still uncertain. Toxins $A$ and $B$ cause structural changes of the cytoskeleton of enterocytes, which results in their shedding and a local inflammatory reaction in response. In the most severe cases, CDAD will cause micro ulcerations covered with mucous from shed epithelial cells $[12,16,17]$. In the pathogenesis of CDAD many cytokines also have an important role, mainly IL-8, but IL-1 $\beta$, IL-6, TNF, IFN- $\gamma$ and leukotriene B4 are often present $[18,19]$. It is not clear what pathogenic factors contribute to CDI in patients with IBD. Presumably it is the result of a weakening of the immune response in the setting of a chronic inflammatory disease, localised compromise of the barrier function of the GI tract, immunosuppression as a result of treatment of the primary disease and disorders of the intestinal flora.

\section{The clinical picture}

For more than 30 years ago, La Mont and Thraka pointed to the importance of CDI in exacerbations of IBD [20]. Just as in the general population, the clinical picture of $C D I$ in patients with IBD may vary from asymptomatic carriers, with various severity of diarrhoea to the most severe, life-threatening forms of colitis. It is often difficult to distinguish between CDI and an IBD exacerbation. Moreover, patients with IBD tend to have an unusual presentation of CDAD: for example diarrhea with blood in a young person, not hospitalized before. These patients undergo more frequent endoscopy and surgical procedures, and are hospitalised more frequently with longer hospital stays, both of which are risk factors for CDI. The CDAD in patients with IBD is characterised by more severe disease course and higher mortality compared with the general population [12]. The inflammatory process usually involves the colon, but may include the small intestine, particularly in patients post colectomy, as well as those who have inflammation of the reservoir (pouchitis) [3, 4].

\section{Recognition}

The CDI diagnosis is based on the detection of Clostridium difficile toxins directly in the stool, most often by ELISA. This is a low-cost test characterised by short execution time (about $1-2 \mathrm{~h}$ ), sensitivity of $75-85 \%$ and a specificity of $95-100 \%$ in the general population. In patients with IBD, ELISA has a lower efficacy, reaching $54 \%$ in the Ananthakrishnan et al. study [9]. The gold standard for diagnosis is a bacterial cytotoxicity assay (CYTA cytotoxin assay test) under culture conditions; however, it is rarely performed because of its complexity and a much longer test time (48-72 h). Other tests available include: breeding Clostridium difficile aimed mainly for epidemiological reasons, tests to detect Clostridium difficile antigens, and PCR. Another EIA test is based on the detection of glutamine dehydrogenase (GDH), a metabolic enzyme that is produced almost exclusively by Clostridium difficile. The test has good sensitivity but poor specificity because it detects colonisa- 
tion of Clostridium difficile. To improve CDAD diagnosis the GDH test can be combined with an ELISA-toxin test. If the test for $\mathrm{GDH}$ is negative the specimen is negative, if both tests are positive the specimen is positive, if the $\mathrm{GDH}$ test is positive and ELISA-toxin test negative an alternative test, such as cytotoxin or PCR test, is required $[21,22]$.

In patients with IBD, diagnostic tests should be carried out with suspicion of CDAD each time there is worsening of the underlying disease $[12,23]$. Screening for CDI in patients without symptoms is not recommended in the general population, which is currently the same recommendation as for patients with CDI. Although it still requires further evaluation, there is more evidence that even patients with ulcerative colitis in remission experienced episodes of diarrhoea with blood [4].

Endoscopy has low diagnostic sensitivity in the diagnosis of CDAD; much lower in IBD than in the general population [3]. Ben-Horin et al. showed pseudomembranes in $50 \%$ of patients with CDI in the general population, while only $13 \%$ of patients with IBD had pseudomembranes. This test remains an important diagnostic tool in the event of complications, unless contraindications are present [24]

\section{Treatment}

The optimal treatment of patients with CDI and $\mathrm{IBD}$, and especially in the exacerbation of symptoms of IBD, has not been clearly defined. It is not established whether the medical treatment of IBD should be continued or modified, especially when concerning immunomodulatory drugs [3]. Treatment similar to that of the general population is indicated in these cases, but its effectiveness is different in patients with IBD. Treatment of mild to moderate CDAD in the general population consists of $500 \mathrm{mg}$ of metronidazole orally every $8 \mathrm{~h}$ for 10-14 days, or 125-500 mg of vancomycin every $6 \mathrm{~h}$ for 10-14 days [3]. In severe infection, the drug of choice is vancomycin at doses of up to $2 \mathrm{~g}$ per day. In the event of complications, oral vancomycin is recommended (dosing as above) in combination with $500 \mathrm{mg}$ intravenous metronidazole every $8 \mathrm{~h}$. In addition, it is recommended to discontinue the antibiotic that contributed to the onset of CDAD and replace it with another if necessary. The cessation of antibiotics causes withdrawal symptoms in up to $25 \%$ of patients in the general population, but patients with IBD have not been separately studied. The efficacy of treatment with metronidazole or vancomycin in the general population is high at $95 \%$ and $99 \%$, respectively $[23,25-27]$. The proportion of unsuccessful therapy with metronidazole in $\mathrm{IBD}$ is higher than in the general population, and only up to $50 \%$ of these patients will be effectively treated with this drug $[7,28]$. Given the more severe CDAD in patients with IBD and the higher risk of complications, many authors recommend the use of vancomycin as first-line drug therapy in patients with a high probability of CDAD in the course of IBD, even before test results are received $[3,10,12]$. Drugs such as rifaximin, bacitracin, nitazoxanide, vancomycin and metronidazole did not have higher efficacy; only teicoplanin had an insignificant increase in efficacy [3, 29]. Data on immunomodulatory treatment are equivocal. Ben-Horin et al. conducted a retrospective, multicentre study, looking at 155 patients with IBD hospitalised for CDAD treated with an antibiotic and an immunomodulator (glucocorticoids, thiopurine, methotrexate, cyclosporine, tacrolimus or biological agents) or just an antibiotic. In $12 \%$ of patients with IBD treated with immunomodulatory drugs, death or colectomy was reported while no such outcomes were observed in the group of patients treated only with antibiotics. Of the assessed immunomodulatory drugs, only cyclosporine showed no negative effects [30]. More recent studies on infliximab also showed no such outcomes [13]. It should be noted, however, that part of the compared studies related to an earlier period, when the role of CDI in patients with IBD was not fully understood, and therefore probably the median time to diagnosis and initiation of therapy was longer and the course of the disease more severe [12]. There is little data on the recurrence of CDAD in patients with IBD. Noteworthy studies have been carried out by Rodemann et al., who showed a lower relapse rate among patients with IBD than in the general population ( $0.1 \%$ vs. $8.7 \%$ ) [31]. Further studies are needed to assess the recurrence of CDI, but it should be noted that when they occur, treatment regimens should be the same as those recommended as in the general population $[25,26]$.

There are promising data on the efficacy of the treatment of patients with IBD using the new antibiotic - fidaxomicin. It is a macrolide antibiotic with a narrow spectrum of bactericidal activity mainly against certain Gram-positive pathogens, characterised by high efficacy against Clostridium difficile and no effect on the physiological bacterial flora of the intestine. The safety of fidaxomicin was proven in two large phase III clinical trials in which more than 1100 patients took part. Study of fidaxomicin, $200 \mathrm{mg}$ orally twice daily, compared with oral vancomycin, demonstrated similiar clinical response and superior sustained responses with a decrease in recurrences (13\% vs. $24 \%$ with vancomycin treatment) $[3,32]$.

The role of probiotics in the treatment and prevention of CDAD in the general population is not fully un- 
derstood. Some studies suggest a positive effect of Saccharomyces boulardii in particular, but these tests lack proper standardisation as they included small patient populations. There are no data on the role of probiotics in the treatment of CDAD in patients with IBD. Perhaps research on monoclonal antibodies will help improve the possibility of preventing CDI recurrence [26, 33, 34].

\section{Summary}

In the last decade the bacterium Clostridium difficile has become one of the most important nosocomial infections. Patients with IBD comprise a special subset of this population. They are characterised by not only a higher risk of developing CDAD due to regular use of immunomodulatory medications and frequent hospitalisations, but they also tend to have a more severe disease course, more frequent complications and higher mortality. In each patient with acute IBD, concomitant CDI should be confirmed or excluded by ELISA. The CDI in patients with IBD should be treated with oral vancomycin $125 \mathrm{mg}$ every $6 \mathrm{~h}$ for 14 days. Although we are starting to get a better understanding of CDI in the course of IBD, there are still many issues that require further study.

\section{References}

1. Hall IC, O'Toole E. Intestinal flora in newborn infants with description of a new pathogenic anaerobe. Am J Dis Child 1935; 49: 390-402.

2. Tedesco FJ, Barton RW, Alpers DH. Clindamycin-associated colitis. A prospective study. Ann Intern Med 1974; 81: 429-33.

3. Navaneethan U, Venkatesh PGK, Shen B. Clostridium difficile infection and inflammatory bowel disease. Understanding the evolving relationship. World J Gastroenterol 2010; 16: 4892-904.

4. Musa S, Thomson S, Cowan M, et al. Clostridium difficile infection and inflammatory bowel disease. Scand J Gastroenterol 2011; 45: 261-72.

5. Ricciardi R, Ogilvie Jr JW, Roberts PL, et al. Epidemiology of Clostridium difficile colitis in hospitalized patients with inflammatory bowel diseases. Dis Col Rect 2009; 52: 40-5.

6. Nguyen GC, Kaplan GG, Harris ML, et al. A national survey of the prevalence and impact of Clostridium difficile infection among hospitalized inflammatory bowel disease patients. Am J Gastroenterol 2008; 103: 1443-50.

7. Issa M, Vijayapal A, Graham MB, et al. Impact of Clostridium difficile on inflammatory bowel disease. Clin Gastroentero Hepatol 2007; 5: 345-51.

8. Issa M, Ananthakrishnan AN, Binion DG. Clostridium difficile and inflammatory bowel disease. Inflamm Bowel Dis 2008; 14: 1432-42.

9. Ananthakrishnan AN, McGinley EL, Binion DG. Excess hospitalization burden associated with Clostridium difficile in patients with inflammatory bowel disease. Gut 2008; 57: 205-10.

10. Ananthakrishnan AN, Binion DG. Impact of Clostridium difficile on inflammatory bowel disease. Expert Rev Gastroenterol Hepatol 2010; 4: 589-600.
11. Banaszkiewicz A, Kowalska-Duplaga K, Pytrus T, et al. Clostridium difficile infection in newly diagnosed pediatric patients with inflammatory bowel disease: prevalence and risk factors. Inflamm Bowel Dis 2012; 18: 844-8.

12. Sinh P, Barrett TA, Yun L. Clostridium difficile infection and inflammatory bowel disease: a review. Gastroenterol Res Pract 2011; 2011 : 136064.

13. Schneeweiss S, Korzenik J, Solomon DH, et al. Infliximab and other immunomodulating drugs in patients with inflammatory bowel disease and the risk of serious bacterial infections. Alim Pharmac Therap 2009; 30: 253-64.

14. Powell N, Jung SE, Krishnan B. Clostridium difficile infection and inflammatory bowel disease: a marker for disease extent? Gut 2008; 57: 1183-4.

15. Clayton EM, Rea MC, Shanahan F, et al. The vexed relationship between Clostridium difficile and inflammatory bowel disease: an assessment of carriage in an outpatient setting among patients in remission. Am J Gastroenterol 2009; 104: 1162-9.

16. Rupnik M, Wilcox MH, Gording DN. Clostridium difficile infection: new developments in epidemiology and pathogenesis. Nat Rev Microbiol 2009; 7: 526-36.

17. Vaishnavi C. Clinical spectrum and pathogenesis of Clostridium difficile associated diseases. Indian J Med Res 2010; 131 : 487-99.

18. Rocha MFG, Maia MET, Bezerra LR, et al. Clostridium difficile toxin $A$ induces the release of neutrophil chemotactic factors from rat peritoneal macrophages: role of interleukin-1beta, tumor necrosis factor alpha, and leukotrienes. Infect Immun 1997; 65: 2740-6.

19. Steiner TS, Flores CA, Pizarro TT, et al. Fecal lactoferrin, interleukin-1beta and interleukin-8 are elevated in patients with severe Clostridium difficile colitis. Clin Diagn Lab Imm 1997; 4: 719-22.

20. La Mont JT, Traka YM. Therapeutic implications of Clostridium difficile toxin during relapse of chronic inflammatory bowel disease. Lancet 1980; 1: 381-3.

21. Bartlett JG. Detection of Clostridium difficile infection. Infect Control Hosp Epidemiol 2010; 31 Suppl 1: 35-7.

22. Simor AE. Diagnosis, management and prevention of Clostridium difficile infection in long-term care facilities: a review. J Am Geriatr Soc 2010; 58: 1556-64.

23. Rahier JF, Ben-Horin S, Chowers Y, et al. European evidence-based consensus on the prevention, diagnosis and management of opportunistic infections in inflammatory bowel disease. JCC 2009; 3: 47-91.

24. Ben-Horin S, Margalit M, Bossuyt P, et al. Prevalence and clinical impact of endoscopic pseudomembranes in patients with inflammatory bowel disease and Clostridium difficile infection. JCC 2010; 4: 194-8.

25. Bricker E, Garg R, Nelson R, et al. Antibiotic treatment for Clostridium difficile-associated diarrhea in adults. Cochrane Database Syst Rev 2007; 3: CD004610.

26. Cohen S, Gerding D, Johnson S, et al. Clinical practice guidelines for Clostridium difficile infection in adults: 2010 update by the Society for Healthcare Epidemiology of America (SHEA) and the Infectious Diseases Society of America (IDSA). Infect Contr Hosp Epidemiol 2010; 31: 431-55. 
27. Gerding DN, Johnson S. Clostridium difficile infection in 2010: advances in pathogenesis, diagnosis and management of CDI. Nat Rev Gastroenterol Hepatol 2011; 8: 67-8.

28. Musher DM, Aslam S, Logan N, et al. Relatively poor outcome after treatment of Clostridium difficile colitis with metronidazole. Clin Inf Dis 2005; 40: 1586-90.

29. Nelson RL, Kelsey P, Leeman $\mathrm{H}$, et al. Antibiotic treatment for Clostridium difficile associated diarrhea in adults. Cochrane Database Syst Rev 2011; 9: CD004610.

30. Ben-Horin S, Margalit M, Bossuyt P, et al. Combination immunomodulator and antibiotic treatment in patients with inflammatory bowel disease and clostridium difficile infection. Clin Gastroenterol Hepatol 2009; 7: 981-7.

31. Rodemann JF, Dubberke ER, Reske KA, et al. Incidence of Clostridium difficile infection in inflammatory bowel disease. Clin Gastroenterol Hepatol 2007; 5: 339-44.

32. Cornely OA, Crook DW, Esposito R, et al. Fidaxomicin versus vancomycin for infection with Clostridium difficile in Europe, Canada, and the USA: a double-blind, non-inferiority, randomised controlled trial. Lancet Infect Dis 2012; 12: 281-9.

33. McFarland LV, Elmer GW, Surawicz CM. Breaking the cycle: treatment strategies for 163 cases of recurrent Clostridium difficile disease. Am J Gastroenterol 2002; 97: 1769-75.

34. van Nispen tot Pannerden CM, Verbon A, Kuipers EJ. Recurrent Clostridium difficile infection: what are the treatment options? Drugs 2011; 71: 853-68.

Received: 6.11.2011

Accepted: $\quad 16.10 .2012$ 